\title{
Renal Leiomyosarcoma-A Rare Entity
}

\section{JCR}

\section{Suresh Babu', Atin Singhai ${ }^{1}$, Nuzhat Hussain ${ }^{2}$, Vishwajeet Singh ${ }^{3}$}

From the Department of Pathology ${ }^{1}$ and Urology ${ }^{3}$, King George's Medical University, Lucknow; Department of Pathology², RML Institute of Medical Sciences, Lucknow, India.

\begin{abstract}
:
Primary sarcomas constitute only $1-2 \%$ of malignant renal tumours in adulthood and of these leiomyosarcomas account for $50-60 \%$. We describe here a case of renal leiomyosarcoma in an elderly woman, presenting with abdominal lump, anorexia and weight loss for 3 months. Based on CECT abdomen findings, a presumptive differential diagnosis of sarcomatoid variant of renal cell carcinoma/ renal sarcoma was made. Microscopy reflected a malignant spindle cell neoplasm which showed strong reactivity for smooth muscle actin with negative epithelial markers, thereby confirming the diagnosis of renal leiomyosarcoma. To conclude the entity of renal sarcoma should be taken in to consideration while evaluating renal tumours, particularly in older females, owing to their aggressive nature and low survival rates.
\end{abstract}

Key words: Leiomyosarcoma, Kidney neoplasms, Sarcoma, Anorexia, Weight loss, Diagnosis.

\section{Introduction}

Malignant smooth muscle tumours of the kidney are uncommon and share only $1-2 \%$ of the total adult renal neoplasms [1]. Of these renal leiomyosarcoma is the most common variant and constitute 50$60 \%$ of the total renal sarcomas [2], however reported literature is very scarce. To the best of our knowledge, this is one of the few reported cases from Indian sub-continent.

\section{Case Report}

A 65 years old female attended the Urology OPD after referral from a primary health centre with chief complaints of right sided abdominal lump, anorexia and weight loss for last 3 months. Her physical examination showed an easily ballotable, non-tender mass, which was lobular in shape and firm in consistency. She was also mildly hypertensive with blood pressure approximately around $160 / 100 \mathrm{~mm} \mathrm{Hg}$ on three occasions. Her past history was irrelevant, except for passing of blood in urine a few times. Her hemogram showed anemia with haemoglobin of $8.2 \mathrm{gm} \%$. The biochemical investigations were all within normal range. Urine analysis showed mild hematuria with 4-6 RBCs/ hpf. Urine culture turned out to be negative but cytology revealed a few atypical cells suspicious for malignancy. Computed tomographic scan of abdomen revealed a large, well defined, lobulated, heterogeneously enhancing soft tissue mass arising

\section{Corresponding Author: Dr. Atin Singhai}

Email: atinsinghai@yahoo.com

Received: December 4, 2013 | Accepted: January 12, 2014 | Published Online: January 20, 2014 This is an Open Access article distributed under the terms of the Creative Commons Attribution License (creativecommons.org/licenses/by/3.0)

Conflict of interest: None declared | Source of funding: Nil | DOl: http://dx.doi.org/10.17659/01.2014.0008 
from right kidney with no evidence of metastases or involvement of any major vessels. Subsequently right nephrectomy was performed. Gross examination revealed a well defined tumor mass measuring $18 \times 8 \times 7 \mathrm{~cm}$, arising from left lateral margin of the nephrectomy specimen with cut surface showing areas of trabeculations and whorling with focal areas of necrosis [Fig.1]. Microscopy from the tumor mass showed a malignant neoplasm composed of spindle cells arranged in fascicles and plexiform pattern, with marked nuclear pleomorphism and prominent nucleoli with acidophilic cytoplasm. Sections from the adjacent renal parenchyma did not reveal any neoplastic component apart from changes of chronic tubulo-interstitial nephritis. Major vessels also showed no sign of tumor dissemination. At this stage, an extensive regrossing was performed to look for any epithelial components in the tumor mass, but revealed none by microscopy. Keeping the differential diagnosis of renal leiomyosarcoma verses sarcomatoid variant of renal cell carcinoma, it was decided to employ immunohistochemistry panel comprising of cytokeratin, smooth muscle actin, vimentin along with $\mathrm{Bcl} 2$ and melanocytic markers like HMB 45 to rule out other remote possibilities like renal synovial sarcoma and epitheloid

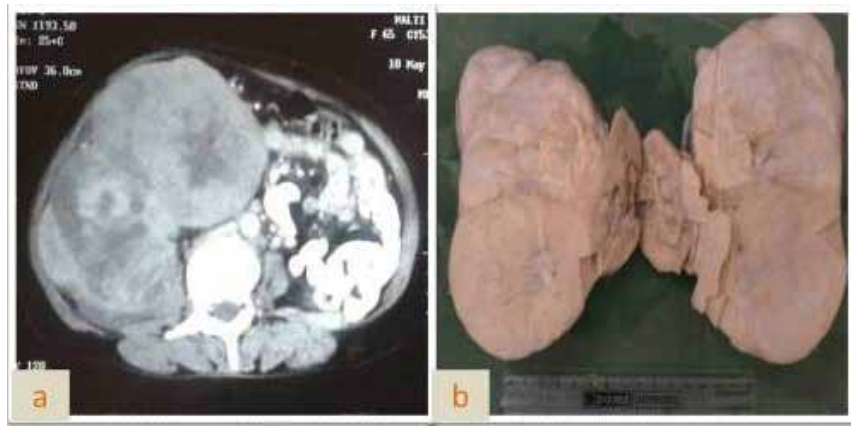

Fig.1: (a) CT abdomen: Well defined lobulated, heterogeneously enhancing, soft tissue attenuation mass arising in the right renal fossa. (b) Gross: Tumor arising from the lateral margins of the nephrectomy specimen with cut surface showing areas of whorling and trabeculations. angiomyolipoma respectively. IHC revealed strong positivity for smooth muscle actin alone while all other markers turned out to be negative [Fig.2]. This established the diagnosis of renal leiomyosarcoma. Moreover, as the renal parenchyma did not reveal any sarcomatous element, it was assumed that this tumor mass originated from the renal capsule. Postoperative recovery of the patient was uneventful and she was advised to go for chemotherapy but she opted out of it. She was discharged after one week with advice for regular follow-up. Till last follow-up approximately 2 months after surgery, the patient was doing well.

\section{Discussion}

As described above, diagnosis of renal leiomyosarcoma was made on microscopy in an elderly female patient of 65 years age presenting with abdominal pain, anorexia and weight loss for 3 months. Main differential diagnosis was kept as

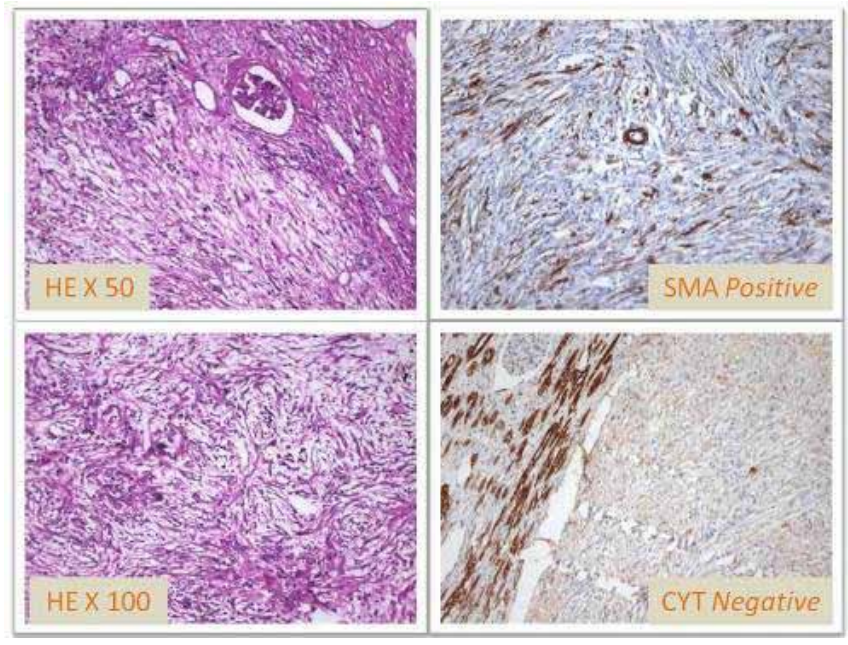

Fig.2: Microscopy revealed a malignant spindle cell neoplasm arranged in fascicles and plexiform pattern with marked nuclear pleomorphism and acidophilic cytoplasm. Renal parenchyma is free from tumour invasion. IHC revealed strong positivity for smooth muscle actin (SMA) while cytokeratin (CYT) was negative. 
sarcomatoid variant of renal cell carcinoma, which was clearly ruled out on immunohistochemistry. The tumor was assumed to be originating from renal capsule, as neither grossly nor microscopically, there was any evidence of sarcomatous elements in renal parenchyma.

Leiomyosarcomas are malignant neoplasms of smooth muscle origin. They are most commonly found in uterus, stomach, small intestine and retroperitoneum [3]. Leiomyosarcomas of renal origin are very rare and constitute only $0.12 \%$ of all malignant renal neoplasms [4]. Histogenesis of renal leiomyosarcomas is believed to be from the renal capsule or the smooth muscle fibres in the renal pelvis or from the renal vessels [5]. They have preponderance in females and are more frequent after 5th decade of life [6].

Renal leiomyosarcomas present with classical clinical triad of abdominal lump, flank pain and hematuria and hence, most of the times, are clinically indistinguishable from primary renal cell carcinomas. Gross examination of the tumor mass may offer some clue in form of evident trabeculations and whorling pattern, but it is the microscopy with immunohistochemistry, which gives the definitive diagnosis. The chief differential diagnosis of renal leiomyosarcoma is with the sarcomatoid variant of renal cell carcinoma. Other differentials which may be considered are epitheloid variant of renal angiomyolipoma and renal synovial sarcoma. Immunohistochemistry is a mandatory tool for evaluation of such tumours to avoid a misdiagnosis [7].

Renal leiomyosarcomas show alternating fascicles of smooth muscle cells with marked nuclear atypia and prominent mitotic figures. They show a positive reaction to smooth muscle markers like smooth muscle actin on immunohistochemistry while the epithelial markers are negative. Sarcomatoid variant of renal cell carcinoma usually reveal a foci of typical renal cell carcinoma and show cytokeratin positivity with negative smooth muscle markers. Epitheloid angiomyolipomas show positivity for smooth muscle as well as melanocytic markers, while renal synovial sarcomas also show positivity for $\mathrm{Bcl} 2$ in addition to smooth muscle markers $[7,8]$.

Renal leiomyosarcomas have an aggressive behaviour owing to rapid growth rate, frequent metastases and high local and systemic recurrence rates. Distant metastases have been reported to lungs, liver and colon. Radical nephrectomy has been considered to offer the best chances of cure. The role of adjuvant chemotherapy/ radiotherapy remains obscure due to paucity of data on treatment of this rare renal neoplasm [9]. Mean survival rate of renal leiomyosarcomas is quite less ranging from 6 months to 2 years after diagnosis $[1,10]$.

\section{Conclusion}

To conclude, histopathological examination with immunohistochemistry is vital diagnostic tool in the diagnosis of renal leiomyosarcomas. The entity of renal leiomyosarcoma should be taken in to consideration while evaluating renal tumours, particularly in older females, owing to their aggressive nature and distant metastases.

\section{References}

1. A Demir, CM Yazici, F Eren, L Turkeri. Case report: Good prognosis in leiomyosarcoma of kidney. Int J Urol Nephrol. 2007;39(1):7-10.

2. Srinivas V, Sogani PC, Hajdu SI, Whiteore WF. Sarcomas of the kidney. J Urol. 1984;132:1316.

3. Weiss SN. Smooth muscle tumours of soft tissue. Adv Anat Pathol. 2002;9(6):351-359.

4. Kendal WS. The comparative survival of renal leiomyosarcomas. Can J Urol. 2007; 14: 34353542.

5. Niceta P, Lavengood RW, Fernandes $M$, Tozzo 
PJ. Leiomyosarcoma of kidney: review of the literature. Urology. 1974;3:270-273.

6. Farrow GM, Harrison EG, Remine WH. Sarcomas and sarcomatoid and mixed malignant tumours of the kidney in adults. Cancer. 1968;22(3):545550.

7. Deyrup AT, Montgomery E, Fisher C. Leiomyosarcoma of the kidney: a clinicopathological study. Am J Surg Pathol. 2004;28(2):178-182.

8. Mai KT, Perkins DG, Collins JP. Epitheloid cell variant of renal angiomyolipoma. Histopathology. 1996;28(3):277-280.

9. Vogelzang NJ, Fremgen AM, Guinan PD, Chmiel JS, Sylvester JL, Sener SF. Primary renal sarcoma in adults: A natural history and management study by the American Cancer Society, Illinois division. Cancer. 1993;71(3):804-810.

10. Sobti P, Rakheja G, Mittal A, Khurana N, Aggarwal SK. Isolated Renal Mucormycosis in a Pediatric Patient. Journal of Case Reports. $2013 ; 3(2): 390-392$. 